\title{
High Speed and Sensitive X-ray Analysis System with Automated Aberration Correction Scanning Transmission Electron Microscope
}

\author{
Hiromi Inada*, Yoichi Hirayama, Keiji Tamura, Daisuke Terauchi, Ryoji Namekawa, \\ Takeharu Shichiji, Takahiro Sato, Yuya Suzuki, Yoshihiro Ohtsu, Keitaro Watanabe, \\ Mitsuru Konno, Hiroyuki Tanaka, Koichiro Saito, Wataru Shimoyama, \\ Kuniyasu Nakamura, Kazutoshi Kaji, Takahito Hashimoto \\ Division of Science \& Medical Systems Design, Science \& Medical Systems Business Group, \\ Hitachi High-Technologies Corporation, Hitachinaka 312-0033, Japan
}

*Correspondence to: Inada $\mathrm{H}$,

Tel: $+81-29-354-5486$

Fax: +81-29-275-5199

E-mail: hiromi.inada.yd@hitachi-hightech.com

Received March 3, 2015

Revised March 24, 2015

Accepted March 24, 2015

\begin{abstract}
We have developed a new HD-2700 (Hitachi High-Technologies Corp., Japan) scanning transmission electron microscope (STEM) that includes an automatic aberration correction function, and a large-solid-angle energy-dispersive X-ray spectroscopy detector that enables high-resolution and sensitive analysis. For observation with atomic resolution, using spherical-aberration-corrected STEM, in order that satisfactory performance of the device can be achieved readily, and within a short time, irrespective of the operator's skill level, a spherical-aberration-correction device with an automatic aberration-correction function was developed. This automatic aberration-correction function carries out the entire correction-related process (aberration measurement, selection and correction) automatically, with automatic selection of the aberrations that require correction, and automatic measurement of the appropriate corrections.
\end{abstract}

Key Words: Aberration correction, Scanning transmission electron microscope, X-ray analysis

\section{INTRODUCTION}

In recent years, the aberration-correction technique has brought a revolution in analytical microscope by making atomic-resolution imaging and analysis routinely achievable in transmission electron microscope (TEM) and scanning transmission electron microscope (STEM). We have developed as a product an electron microscope the performance of which is dramatically increased by inclusion of a sphericalaberration-correction function (Inada et al., 2009a, 2009b). In addition, the application of new aberration-correction techniques, such as atomic-resolution secondary-electron (SE) imaging, is now being investigated (Zhu et al., 2009; Inada et al., 2011a, 2011b; Inada \& Zhu, 2014).

Scherzer (1947) proved that combinations of rotationally symmetrical electromagnetic lenses had convex lens effects only, and the spherical aberration coefficients were always positive. However, multipole lenses in the aberrationcorrection devices of TEMs and STEMs have resulted in concave lens effects, that is, lenses with negative spherical aberrations, and these are now in wide use for cancelling out the positive spherical aberrations of object lenses (Beck, 1979; Rose, 1981; Crewe, 1982; Rose, 1990; Haider et al., 1998). On the other hand, optics systems using multipole lenses give rise to various types of parasitic aberration due to the heterogeneity of the magnetic properties of the materials, and slight deviations from symmetry during machining. With the aberration-correction devices in previous use, for correcting multiple types of aberration, alignment was a difficult process, and users required considerable experience to be able to make

(a) This is an open-access article distributed under the terms of the Creative Commons Attribution Non-Commercial License (http://creativecommons.org/licenses/by-nc/3.0) which permits unrestricted noncommercial use, distribution, and reproduction in any medium, provided the original work is properly cited.

Copyrights @ 2015 by Korean Society of Microscopy 


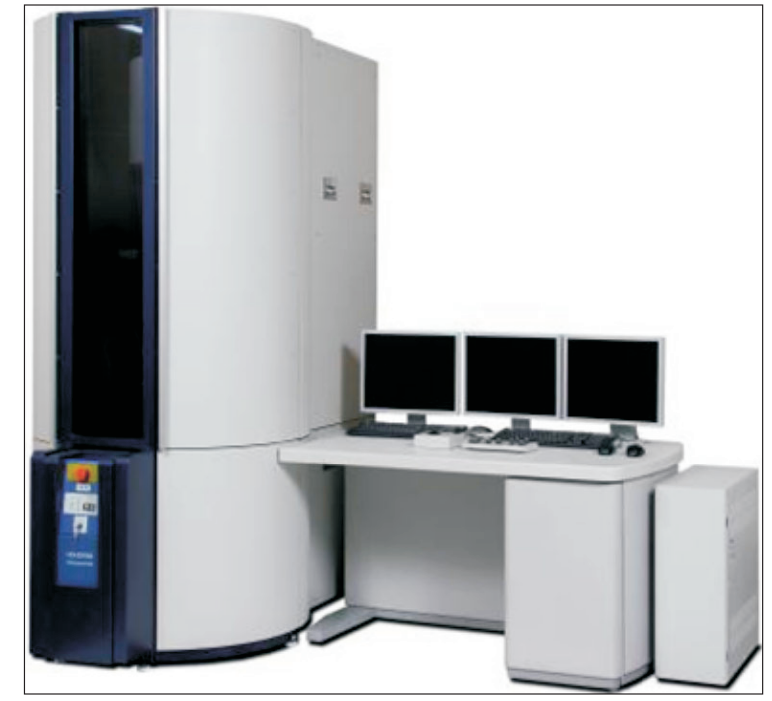

Fig. 1. External appearance of HD-2700 (Hitachi High-Technologies Corp.) scanning transmission electron microscope that includes an automatic aberration-correction function as well as large solid angle silicon drift detectors.

the appropriate adjustments for achievement of satisfactory performance within a reasonably short time. We have recently developed a spherical-aberration-corrector that includes an automatic aberration-correction function enabling atomic-level high-resolution imaging by means of a simpler operation, so that satisfactory performance of the microscope can be achieved more readily, irrespective of the user's skills and experience.

From the analytical capability point of view, for studies using STEM on the nanometer scale, energy-dispersive X-ray spectroscopy (EDX) is in widespread use. However, the EDX detectors in previous use were insufficiently sensitive, and did not enable elemental mapping within a short time. In order to achieve sensitive X-ray spectroscopic signals, it is essential to increase the solid angle and sensitivity of detection. The present report relates to the recently developed HD-2700 (Hitachi High-Technologies Corp., Japan; Fig. 1), to which a silicon drift detector (SDD) with a large detection area for high-resolution X-ray analysis can be attached.

\section{CONFIGURATION OF THE ABERRATION- CORRECTION SYSTEM}

\section{HD-2700 Analytical CFEG-STEM}

Fig. 2 shows a schematic diagram of the HD-2700. It includes a cold-field emission electron gun, and can be operated at accelerating voltages of 80,120 , or $200 \mathrm{kV}$. The electronbeam-probe spherical-aberration-correction device in this device is positioned between the condenser lens system and the object lens. The cold field electron source gives high- brightness and small energy spread. Fig. 3 shows an electron beam energy distribution, measured with an electron energyloss spectroscopy (EELS) detector (Gatan Inc., USA) indicates energy spread of $0.35 \mathrm{eV}$ full-width half-maximum for acquisition time of one second. That allows users to acquire high energy resolution spectra with EELS.

The Everhart-Thornley SE detector is attached in the upper part of the object lens pole-piece, and bright-field (BF) and annular dark-field (ADF) STEM detectors are set below the specimen. The six electron micrographs indicated by Fig. 2A-F are examples of observation of metal nanoparticles at low and high magnification recorded by each of the SE, ADF-STEM and BF-STEM detectors. With BF-STEM, a phase-contrast image corresponding to the TEM image was obtained. Darkfield (DF)-STEM imaging involves detection of high-angle (HA) scattered electrons, and is also termed " $\mathrm{Z}$-contrast imaging", because the contrast is proportional to the square of the atomic number, the atomic number symbol being " $\mathrm{Z}$ ". This technique enables generation of localized composition information about the specimen. SE imaging reflects the superficial structure of the specimen, and one characteristic is that it enables observation of the three-dimensional structure of the specimen. In Fig. 2D is an example of a photograph of the atomic column in metal, obtained by SE (Inada et al., 2011a). High-resolution observation by SE enables direct determination of the supporting states of catalyst particles, etc. The HD-2700 is equipped with a charge-coupled device (CCD) camera that can be retracted out of the optical axis (Fig. $2 \mathrm{H}$ ), and there is the option of including an EDX (Fig. 2I) and/or EELS detector, either the EV3000 elements view (Hitachi High-Technologies Corp.) (Kaji et al., 2001; Isakozawa et al., 2008) or the Enfinium (Gatan Inc.), which are used for elemental analysis. EV3000 is a sort of scanning filter image detector allows to obtain real-time elemental mapping with DF-STEM image simultaneously (Fig. 2G). EV3000 has two kinds of detector, one is for spectrum and another is three-channel photo-multipliers for direct element observation using an electro-magnetic prism (Kaji et al., 2001; Isakozawa et al., 2008).

In addition to STEM imaging, the HD-2700 including an aberration-correction device performs SE imaging with atomic-level resolution, and also elemental analysis, mapping of the chemical bond statuses, etc., it can collect information from specimens in a versatile manner, and it dramatically improves analysis performance (Zhu et al., 2009; Inada et al., 2011a, 2011b; Inada \& Zhu, 2014).

\section{Automatic Aberration-correction Function}

The optics system of the spherical-aberration-correction device developed for the HD-2700 is constructed of multipole and transfer lenses. This aberration-correction device is positioned on the upper part of the object lens, and gives 

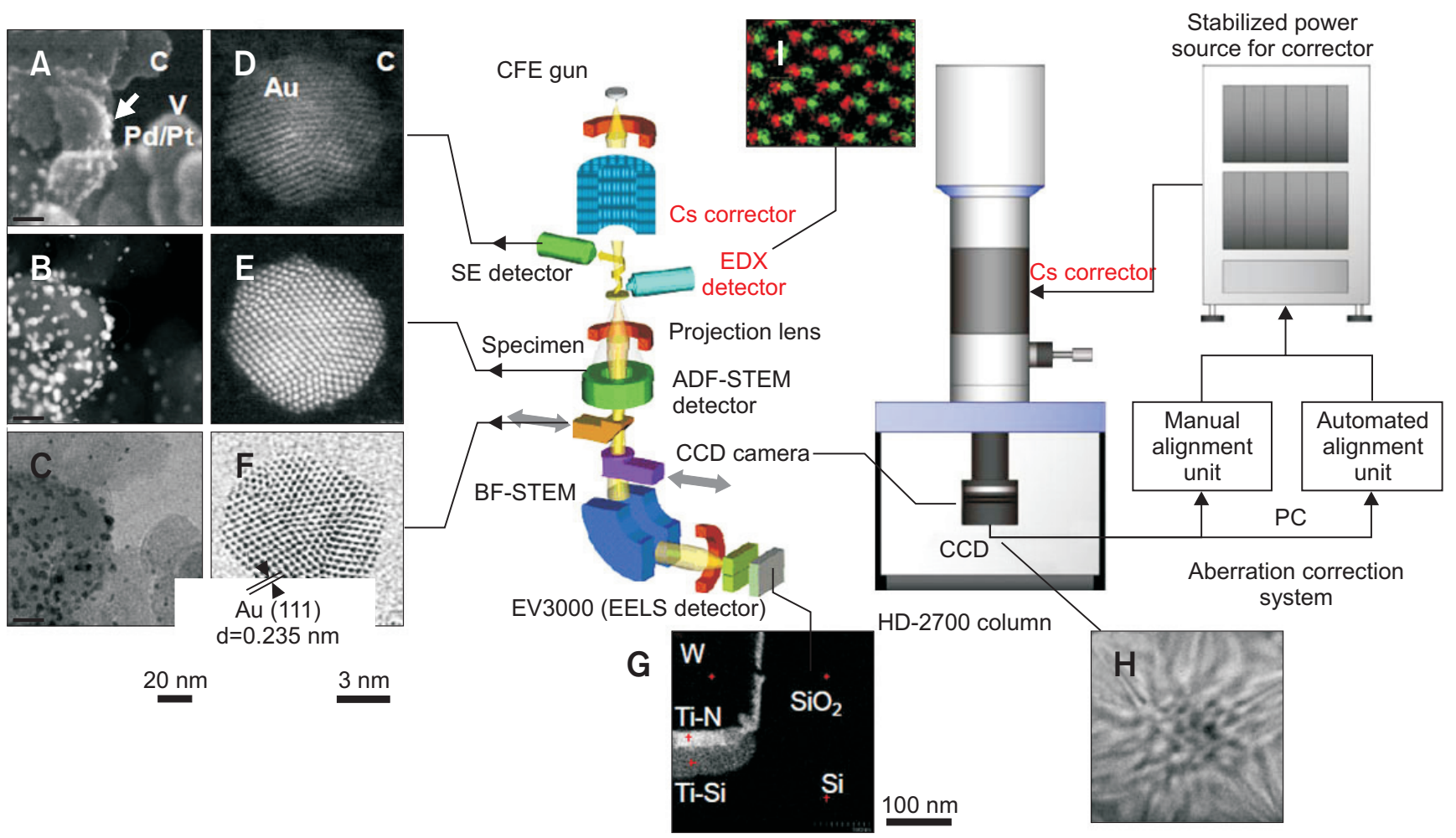

Fig. 2. Schematic diagram of the HD-2700 detector (Hitachi High-Technologies Corp.), and comparison of the images obtained with each detector and system of the automatic aberration-correction system (the object lens is omitted from this diagram). (A-C) SE, ADF-STEM and BF-STEM images of Pd/Pt catalyst powder on a carbon support. (D-F) Atomic-resolution SE, ADF-STEM and BF-STEM images of gold nanoparticles deposited on a thin carbon film. Photogram ' $G$ ' shows an example of elemental image of silicon deveice close to the tungsten (W) plug taken with Hitachi EV3000, 'H' shows a Ronchigram of amorphous thin film and 'I' shows an atomically resolved GaAs column by column X-ray mapping with Oxford SDD detector. C, carbon support; V, vacuum; Pd/Pt, palladium/platinum; Au, gold nanoparticles; CFE gun, cold-field emission electron gun; SE detector, secondary-electron detector; BFSTEM, bright-field scanning transmission electron microscope; EELS detector, electron energy-loss spectroscopy detector; Cs, spherical aberration coefficient; EDX detector, energy-dispersive X-ray spectroscopy detector; ADF-STEM, annular dark-field STEM; CCD camera, charge-coupled device camera; PC, personal computer.

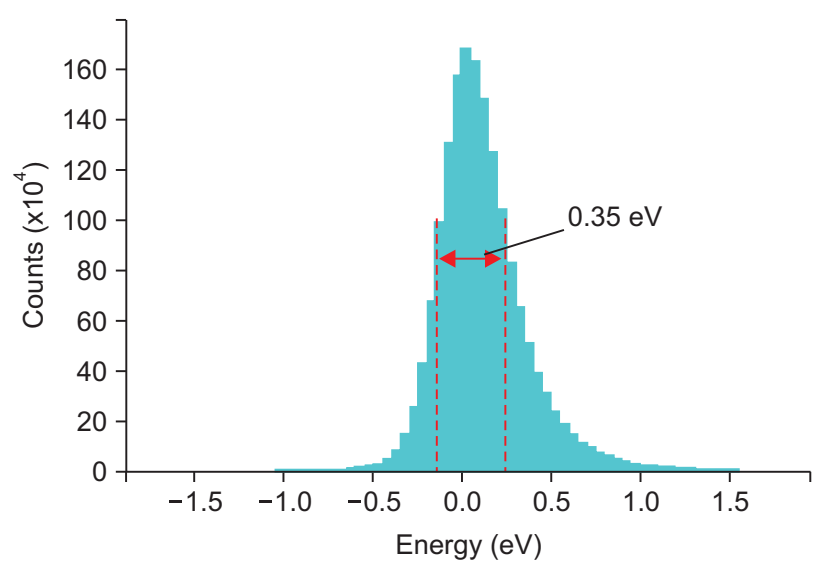

Fig. 3. Electron beam energy distribution of cold-field emission electron gun on HD-2700 (Hitachi High-Technologies Corp.) measured with Gatan electron energy-loss spectroscopy detector (Gatan Inc.; Acceleration voltage: $200 \mathrm{kV}$, acquisition time: $1 \mathrm{~s}$, dispersion: $0.05 \mathrm{eV} /$ pixel). rise to a negative spherical aberration sufficient to cancel out the positive spherical aberration of the object lens. Without spherical aberration correction, the spherical aberration resulted in HA components of the electron beam being focused at different positions from low-angle components, so the electron beam did not converge at a single point. When the equipment is fitted with a spherical-aberration-correction electron beam that has HA components with large off-axis distances are focused on the Gaussian plane, not only the image resolution is increased, but also the probe current can also be increased because of using larger aperture bore due to increasing of irradiation angle.

In this optics system, seven types of aberration are targets for correction, these being first- to third-order aberrations, including spherical aberration, and astigmatism and other residual aberrations. The automatic aberration-correction system can correct these aberrations effectively, and in the optimal sequence. Fig. 2 on the right schematic shows an overview of the automatic aberration-correction system 
included with HD-2700. Aberration correction is based on the results of aberration measurement, and aberration measurement involves obtaining a Ronchigram of an amorphous specimen using a CCD camera, and then calculating each aberration coefficient by image processing. The correction software automatically determines the type and magnitude of the corrected aberration, on the basis of the aberration coefficient obtained, provides feedback to the power source, to control the aberration-correction lens and alignment coils. For each aberration to be corrected, these procedures are repeated automatically until the level of blur has been reduced to no more than a specified value. One can choose manual alignment mode as well as the auto function. For the power supply, high-precision electronics parts are selected and the circuit is designed in such a manner that the power source stability is less than $1 \mathrm{ppm}$.

Fig. 4 shows images of a single-crystal-silicon $<110>$ thinfilm specimen, obtained by HAADF-STEM, before and after automatic aberration correction. In the line profiles of the HAADF-STEM images, there are dumbbell at an interval of $136 \mathrm{pm}$, and whereas the image obtained before automatic aberration correction shows only a small trough, and an illdefined shape, the image obtained after automatic aberration correction is much clearer. It is therefore considered that automatic aberration correction corrects all aberrations sufficiently, and focuses the electron beam. The spot corresponding to the $105 \mathrm{pm}$ point was confirmed in the fast-Fourier-transform image after auto-correction. The time
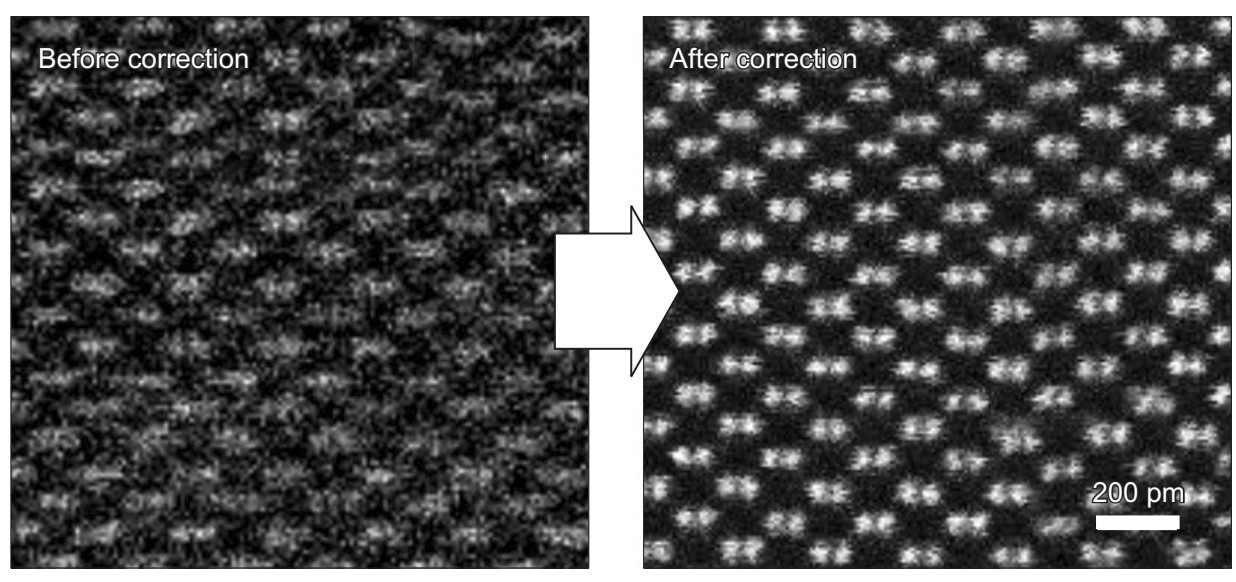

Fig. 4. High-resolution high-angle annular dark-field scanning transmission electron microscope images of a single-siliconcrystal $<110>$ thin-film, before and after automatic aberration correction.
A

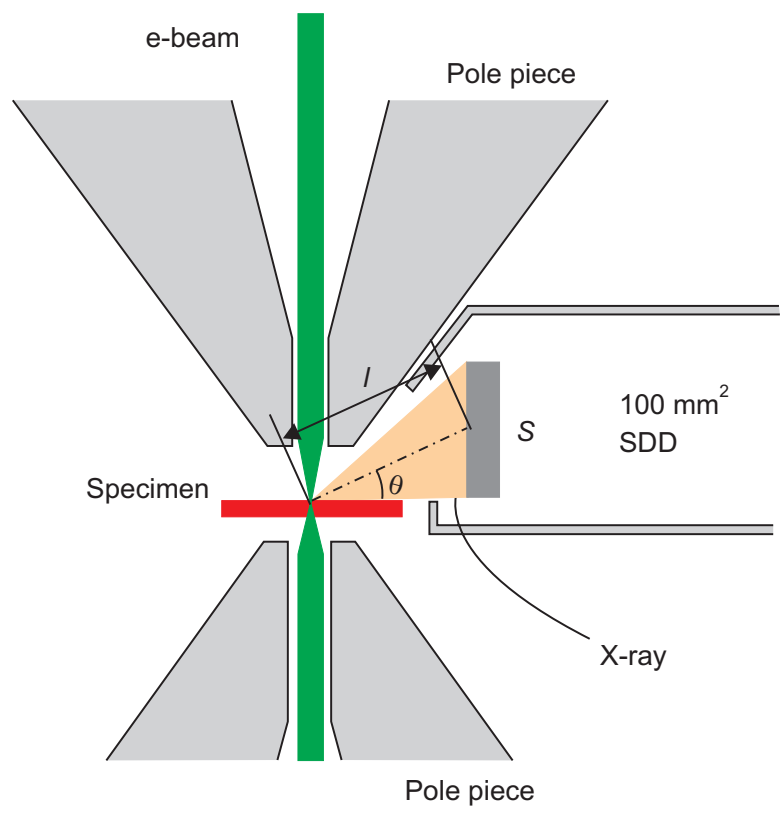

B

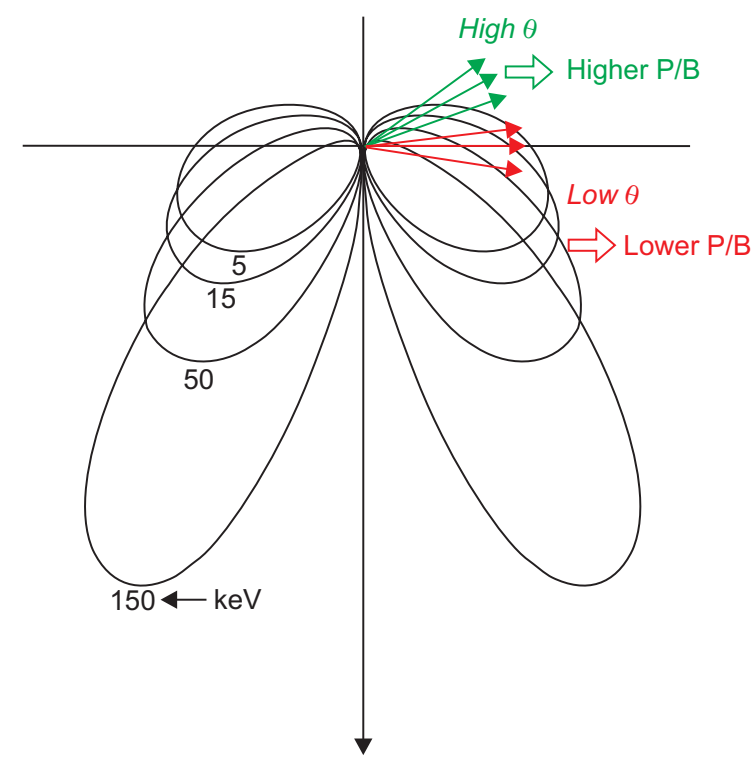

Fig. 5. Relationship between the positions of the object lens and silicon drift detector (SDD) detector (A) and Sequential X-ray emission and angular distribution (B). l, distance (mm); S, active area of the sensor $\left(\mathrm{mm}^{2}\right)$; $\theta$, take off angle $\left({ }^{\circ}\right)$; P/B, peak/background. 
required for automatic correction varies, dependent upon the aberration remaining at initiation, but it is completed within several to 30 minutes.

\section{INCREASING X-RAY DETECTION SENSITIVITY}

Achieving high-sensitive X-ray analysis, it is essential to increase solid angle of the EDX detector. Fig. 5 shows simplified

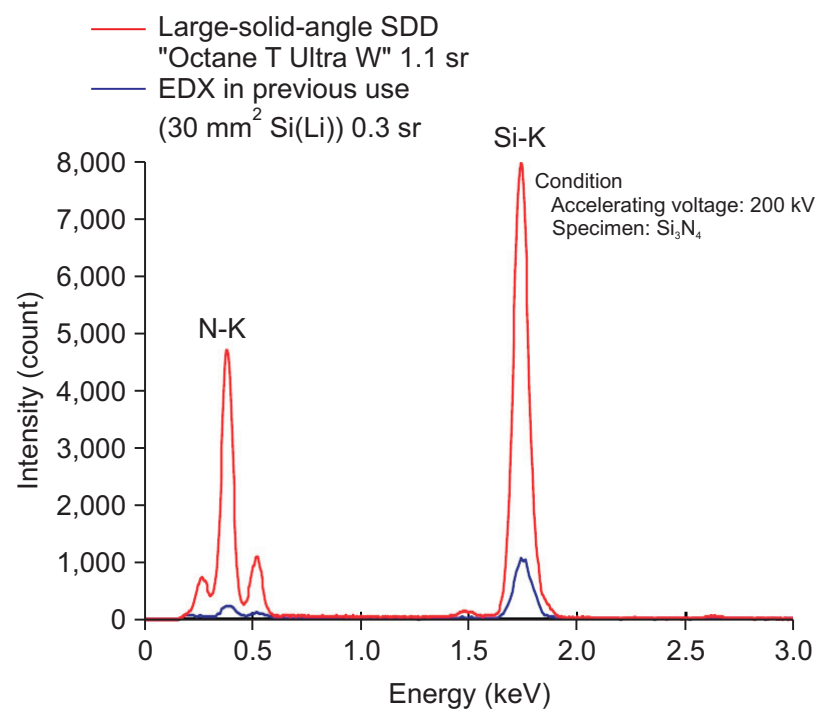

Fig. 6. Differences between spectra with the previous silicon/lithium detector and the newly developed large-solid-angle silicon drift detector (SDD). N-K, N K-edge; Si-K, Si K-edge; EDX, energy-dispersive X-ray spectroscopy. schematics of an EDX detector around the objective lens polepiece.

The detection efficiency of an EDX detector is evaluated based on the solid angle, $\Omega$ is given by

$$
\Omega=\frac{S \cos \theta}{l^{2}}
$$

where $S$ is the active area of the sensor $\left(\mathrm{mm}^{2}\right), \theta$ is the take off angle (TOA) (degrees) of the detector from the specimen, and 1 is the distance $(\mathrm{mm})$ from the specimen to the detector (Zaluzec, 2014). To improve the X-ray detection efficiency of the EDX system, the following points must be taken into consideration: 1) Selecting SDD sensor with a large active area of S, 2) Optimization of the objective lens pole piece design with the SDD geometry with shorter distance between specimen and sensor 1 and higher TOA $\theta, 3$ ) Using a windowless design to eliminate counting loss of X-ray signal especially for low energy signal, and 4) Multiple detector assignment around the sample geometrically optimized to the detector.

Note that Zaluzec (2014) recently suggested that the geometry of the specimen and detector should be considered more strictly for current large solid angle X-ray detector design because of the critical for the distance between the specimen and the sensor, which makes relatively smaller solid angle compared to the numbers derived by the equation (1). As a result of the suggested solid angle can be reduced approximately $20 \%$ smaller than that of calculated with equation (1).
A
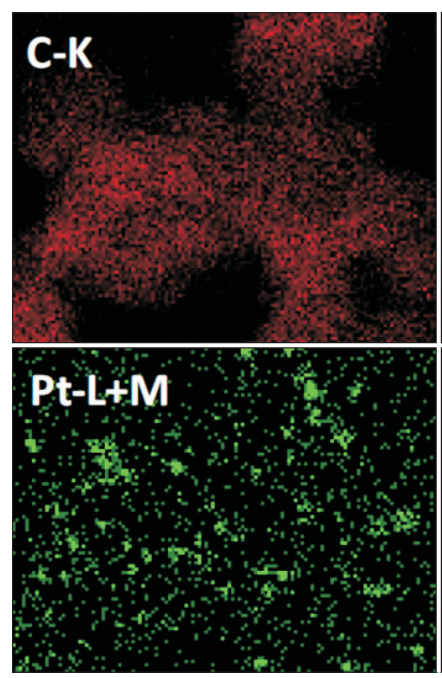
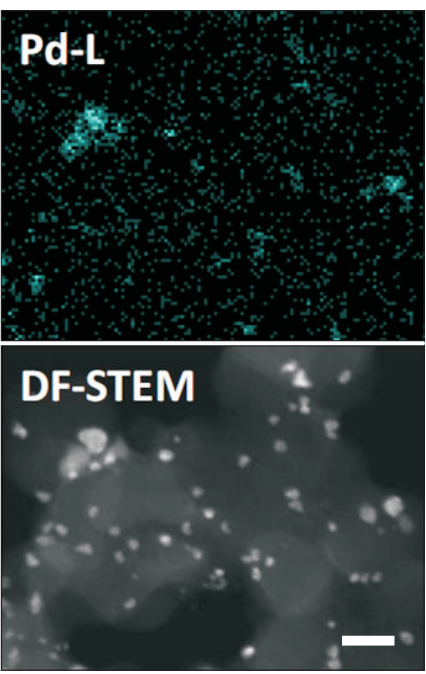

B
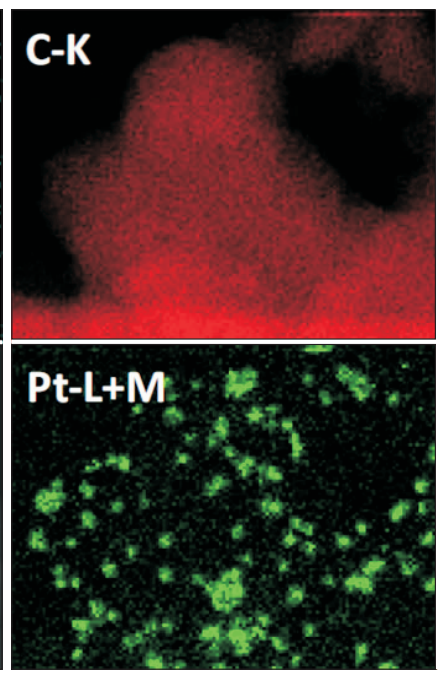
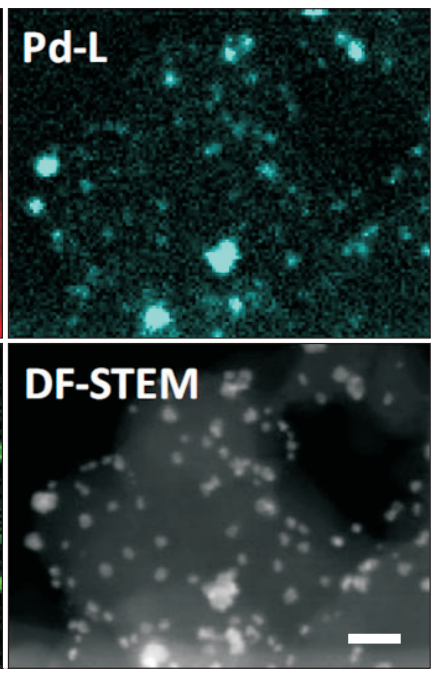

Fig. 7. (A) Comparison of energy-dispersive X-ray spectroscopy mapping images of palladium/platinum catalyst particles. (A) 30 mm ${ }^{2} \mathrm{Si}(\mathrm{Li})$ detector in previous use; count rate: $~ 3,000$ cps. (B) Newly developed $100 \mathrm{~mm}^{2}$ silicon drift detector "Octane T Ultra W"; count rate: $35,000 \mathrm{cps}$. C-K, C K-edge; Pd-L, Pd L-edge; Pd-L+M, Pd L-edge+M-edge; DF-STEM, dark-field scanning transmission electron microscope. Scale bars=20 nm. 
We have developed two large solid angle EDX detectors collaborating with EDAX Inc. (USA) and Oxford Instruments (UK). As described, we applied two different possible approaches. Applying 1) to 3) for EDAX one (in the section "A Large-Solid-Angle and Windowless X-ray Detector") and 1) to 4) with Oxford one (in the section "Multiple SDDs for Increasing Sensitivity").

\section{A Large-Solid-Angle and Windowless X-ray Detector}

\section{Configuration}

The newly developed SDD, Octane T Ultra W (EDAX Inc.), is included with the HD-2700, for high-sensitivity elemental analysis. For achievement of large solid angles, and effective acceptance of incident X-rays, a large SDD element, with an element surface of $100 \mathrm{~mm}^{2}$, is used (Tamura et al., 2013). The shape of the pole-piece was designed so that the detector approaches the specimen closely, and a large X-ray take-off solid angle (1.1 sr) is achieved, as shown in Fig. 5A. The characteristic X-rays emitted by the specimen radiate isotropically, but, as shown in Fig. 5B, the continuous X-rays show an angular distribution (Fiori et al., 1986). Therefore, the option of increasing the TOA, $\theta$, when deciding upon the configuration and positioning of the SDD elements, in order to increase the peak/background ratio of the X-ray spectrum, is being examined.

Characteristics of this SDD are that it uses the drift electrode to concentrate electric charge on minutely small contacts, followed by output, so the capacitance is decreased; the energy resolution is not decreased even when the X-ray incidence rate is high; and analysis is performed at high speed. In addition, inclusion of a cooling step using a Peltier element renders unnecessary the inclusion of liquid nitrogen, which was inconvenient and time-consuming to replace, thus enabling measurement 5 minutes after initiation of cooling.

This SDD maintains energy resolution at no higher than 132 $\mathrm{eV}$, which is approximately the same as with the $30 \mathrm{~mm}^{2} \mathrm{SDD}$ in previous use. The aim was to remove the ultra-thin window (UTW) film on the front face of the EDX detector element in previous use, to make the device windowless, and this resulted in elimination of X-ray absorption by the UTW film and its supporting structure, thus increasing the detection sensitivity of the light element (Isakozawa et al., 2010).

\section{Results of measurements and application}

Fig. 6 shows the results of comparison of the STEM-EDX spectrum X-ray detection sensitivity with silicon nitride $\left(\mathrm{Si}_{3} \mathrm{~N}_{4}\right)$ thin-film specimens, at $200 \mathrm{kV}$, between the $30 \mathrm{~mm}^{2}$ UTW-type $\mathrm{Si}(\mathrm{Li})$ detector in previous use, and the $100 \mathrm{~mm}^{2}$ SDD now developed. The sensitivity was found to be higher with the $100 \mathrm{~mm}^{2}$ SDD, and, in particular, there was a marked difference in sensitivity on the low-energy sides to the peaks.
In the case of nitrogen (the $\mathrm{N}-\mathrm{K}$ peak), for example, the sensitivity with the two devices was found to differ by at least an order of magnitude, showing the differences between the characteristics of the windowless and large-solid-angle detectors. For elemental mapping of catalyst particles in the palladium/platinum $(\mathrm{Pd} / \mathrm{Pt})$ core-shell structure supported on carbon, Fig. 7 shows the results of comparison of the above detector types, with a probe current of $800 \mathrm{pA}$, and an acquisition time of 3 minutes. The shape of all nanoparticles was more clearly defined when using the $100 \mathrm{~mm}^{2}$ detector. With the detector in previous use, a longer acquisition time was needed, so the specimens had to be irradiated with the probe current for an extended period, resulting in damage to the specimens. Together with increasing the detection sensitivity, it was found to be possible to reduce the electron dose for the specimens, and to carry out mapping in a shorter time.

\section{A}

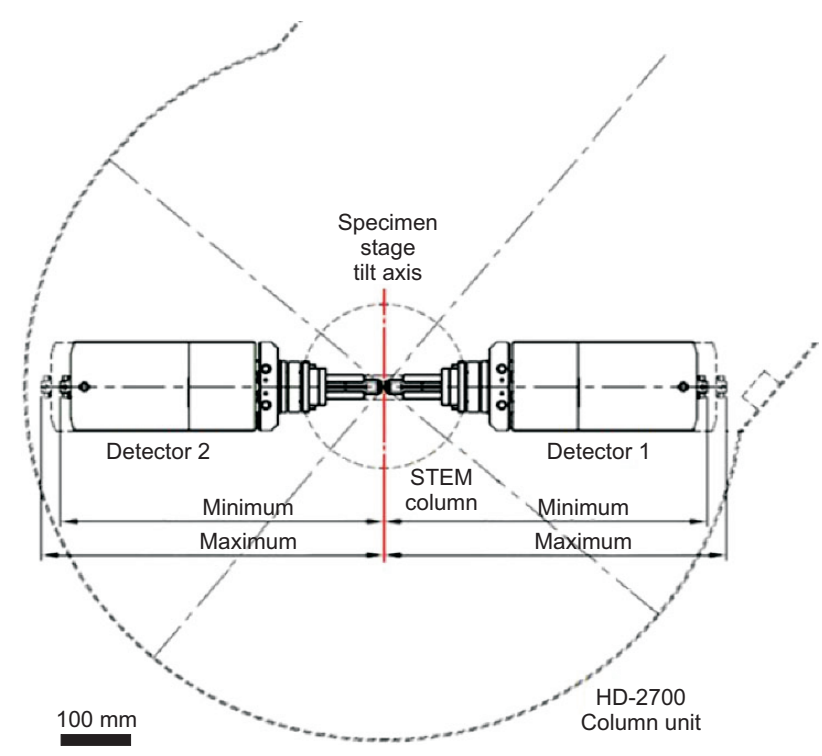

B

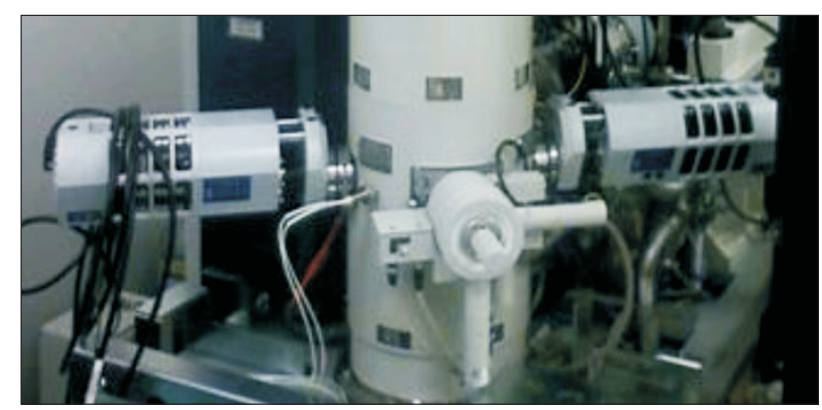

Fig. 8. Configuration of two energy-dispersive X-ray spectroscopy detectors (Oxford Instruments X-MaxN 100 TLE) mounting on HD-2700 (A) and its appearance (B). 
Raw data

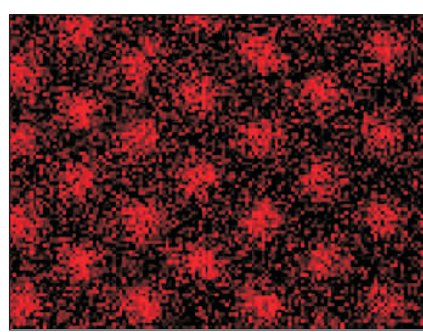

Ga-K

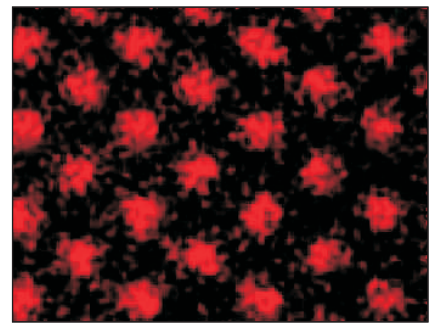

Filtered data

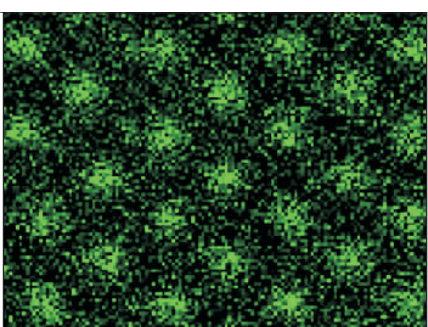

As-K

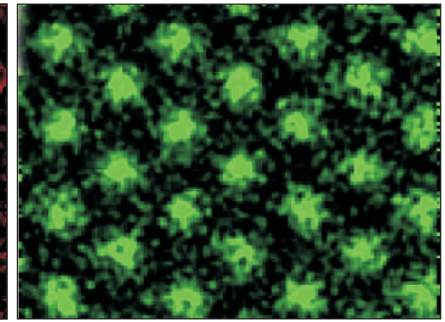

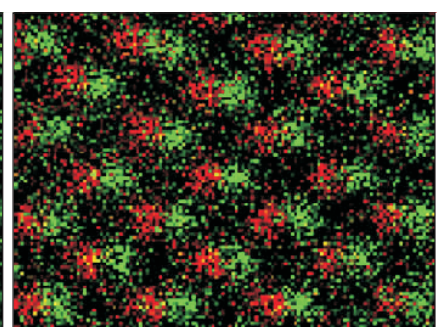

$\mathrm{Ga}+\mathrm{As}$

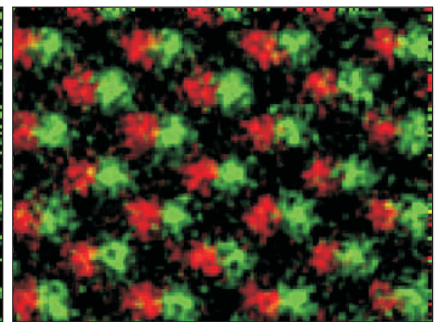

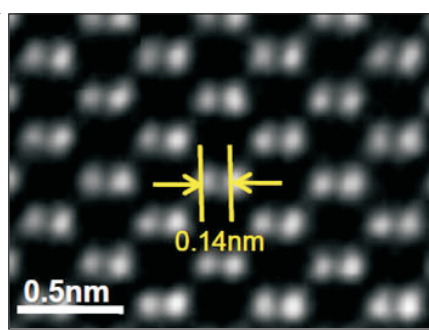

ADF-STEM

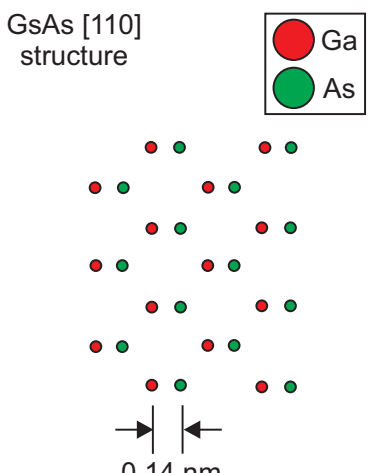

$0.14 \mathrm{~nm}$

Fig. 9. Atomic resolution energy-dispersive X-ray spectroscopy mapping of GaAs (Hashimoto et al., 2014). Acquisition condition: HD-2700 (Hitachi HighTechnologies Corp.) high-resolution pole-piece on acceleration voltage of $200 \mathrm{kV}$. Pixel resolution 128×96 pixel, Dwell time $0.1 \mathrm{~ms} /$ pixel, for $12 \mathrm{minutes}$. Probe current of 45 pA. ADF-STEM, annular dark-field scanning transmission electron microscope.

\section{Multiple SDDs for Increasing Sensitivity}

Another approach to increase sensitivity is to apply multiple EDX detectors surrounding the sample. For this study, we adopt two Oxford Instruments X-MaxN 100 TLE SDDs are mounted to the HD-2700. The SDD exploits a $100 \mathrm{~mm}^{2}$ sensor and a windowless configuration. These features are suitable to take highly sensitive X-ray detection especially for light elements. Due to the sensor design and its geometry, this SDD has a solid angle of $1.04 \mathrm{sr}$ and a TOA of 22.8 degrees each detector for high-resolution pole-piece.

The objective lens was modified to accommodate a second detector at the opposite side to the first detector, with azimuth angle of 90 and 270 degrees to the specimen-tilt axis for the detectors. Fig. 8 shows the dual detector layout. In this system, signals from two separated analyzer can be added electronically and simultaneously so that X-ray count rate is increased approximately by a factor of 2 .

As a result of sensitivity comparison, using a single detector each and dual detector configuration with Nickel Oxide thin foil $(65 \mathrm{~nm})$ specimen on acceleration voltage of $200 \mathrm{kV}$, in case of specimen tilt angle at zero degrees, each detector count rate indicates 20,000 count per second (cps) respectively. And it is revealed 40,000 cps summed up both two detector signals. Although the disadvantageous tilt setting for one detector is compensated by the advantageous tilt condition of the other detector in this geometry, some specimens, such as semiconductor devices, interfaces, or those that require crystallographic orientation may not always be analyzed with optimum tilt condition towards SDD. The dual detector configuration is suitable for such applications.

Dual EDX configuration shown Fig. 8 provides not only increasing sensitivity of X-ray signal but more importantly for atomic resolving column by column elemental mapping to collect zone axis orientation, which can be compensate X-ray signal intensity each other, even one of others reducing the signal opposite direction of the detector. Fig. 9 is an atomic column map of GaAs thin film. The upper row images are raw data and the lower low-pass filtered. Spacing of Ga and As column is $0.14 \mathrm{~nm}$ (Hashimoto et al., 2014).

\section{CONCLUSIONS}

We have developed a new HD-2700 STEM that includes an automatic aberration correction function, and a large-solidangle EDX detector that enables high-resolution and sensitive analysis.

For observation with atomic resolution, using sphericalaberration-corrected STEM, in order that satisfactory performance of the device can be achieved readily, and within a short time, irrespective of the operator's skill level, a spherical-aberration-correction device with an automatic aberration-correction function was developed. This 
Inada $\mathrm{H}$ et al.

automatic aberration-correction function carries out the entire correction-related process (aberration measurement, selection and correction) automatically, with automatic selection of the aberrations that require correction, and automatic measurement of the appropriate corrections. This device makes it possible to eliminate selection of aberrations requiring correction, which was the operation within aberration correction that required experience on the part of the operator, so, by automation, simpler and higher-speed operation of the spherical-aberration correction device has been achieved.

Increasing X-ray analysis detection sensitivity, the HD-2700 includes a $100 \mathrm{~mm}^{2}$ windowless and high TOA SDD with a large solid angle ( $>1 \mathrm{sr}$ ) ensure the higher detection efficiency. In comparison with the device in previous use, a UTW-fitted $30 \mathrm{~mm}^{2} \mathrm{Si}(\mathrm{Li})$ detector, the sensitivity with this SDD has been increased by at least an order of magnitude. In connection with improving the detection sensitivity, high-speed, highsensitivity X-ray analysis can be achieved, and this device thus also enables mapping without damage due to the electron beam. In the case of dual EDX detector configuration, not only double the nominal solid angle, this configuration is suitable for application that requires crystallographic orientation such as atomic resolved column mapping. We expect this system contributes in many fields of research work and failure analysis for nanomaterials.

\section{CONFLICT OF INTEREST}

No potential conflict of interest relevant to this article was reported.

\section{ACKNOWLEDGEMENTS}

We wish to acknowledge developments and support many coworkers: R. Buchhold, B. Hammell and A. Sandborg and Kousuke Rikukawa of EDAX Inc. in U.S.A. and Simon Burgess, James Holland, Iain Anderson, Susumu Yamaguch of Oxford Instruments in U.K.

\section{REFERENCES}

Beck V D (1979) A hexapole spherical aberration corrector. Optik 53, 241 255.

Crewe A V (1982) A system for the correction of axial aperture aberrations in electron lenses. Optik 60, 271-281.

Fiori C E, Swyt C R, and Ellis J R (1986) A critique of the continuum normalization method used for biological X-ray microanalysis. In: Principles of Analytical Electron Microscopy, eds. Joy DC, Romig AD, and Goldstein J, pp. 413-443, (Springer Press, Boston).

Haider M, Rose H, Uhlemann S, Kabius B, and Urban K (1998) Towards $0.1 \mathrm{~nm}$ resolution with the first spherically corrected transmission electron microscope. J. Electron Microsc. 47, 395-405.

Hashimoto T, Tamura K, Inada H, Watanabe K, Ohtsu Y, Suzuki Y, Sato T, Kanemura T, Burgess S, and Holland J (2014) Development of two steradian EDX system for the HD-2700 FE-STEM equipped with dual X-Max N 100 TLE large area windowless SDDs. Microsc. Microanal. 20, 604-605.

Inada $\mathrm{H}$, Kakibayashi $\mathrm{H}$, Isakozawa $\mathrm{S}$, Hashimoto $\mathrm{T}$, Yaguchi $\mathrm{T}$, and Nakamura K (2009a) Chapter 4: Hitachi's development of cold-field emission scanning transmission electron microscopes. In: Advances in Imaging and Electron Physics, ed. W H Peter, Volume 159, pp. 123-186, (Elsevier Press, Burlington).

Inada H, Konno M, Tamura K, Suzuki Y, Nakamura K, and Zhu Y (2011a) Atomic resolution secondary electron imaging in aberration corrected STEM. Microsc. Anal. S5-S8.

Inada H, Su D, Egerton R F, Konno M, Wu L, Ciston J, Wall J, and Zhu Y (2011b) Atomic imaging using secondary electrons in a scanning transmission electron microscope: experimental observations and possible mechanisms. Ultramicroscopy 111, 865-876.

Inada H, Wu L, Wall J, Su D, and Zhu Y (2009b) Performance and image analysis of the aberration-corrected Hitachi HD-2700C STEM. J. Electron Microsc. 58, 111-122.
Inada H, and Zhu Y (2014) Secondary electron microscopy in STEM. In: Scanning Transmission Electron Microscopy of Nanomaterials, ed. Tanaka N, Chapter 10, pp. 307-344, (Imperial College Press, Hackensack).

Isakozawa S, Kaji K, Jarausch K, Terada S, and Baba N (2008) The development and characteristics of a high-speed EELS mapping system for a dedicated STEM. J. Electron Microsc. 57, 41-45.

Isakozawa S, Kaji K, Tamura K, Zhang X F, Sandborg A, and Baba N (2010) The development of a new windowless XEDS detector. J. Electron Microsc. 59, 469-472.

Kaji K, Ueda K, Aoyama T, Taya S, Tanaka H, and Isakozawa S (2001) Development of a real-time jump-ratio imaging system equipped with a STEM. J. Electron Microsc. 50, 15-21.

Rose $\mathrm{H}$ (1981) Correction of aperture aberrations in magnetic systems with threefold symmetry. Nucl. Instrum. Methods 187, 187-199.

Rose H (1990) Outline of a spherically corrected semiaplanatic mediumvoltage transmission electron-microscope. Optik 85, 19-24.

Scherzer O (1947) Spharische und chromatische korrektur von elektronenlinsen. Optik 2, 114-132.

Tamura K, Namekawa R, Buchhold R, Hammell B, Sandborg A, Sato T, Konno M, Inada H, Nakamura K, and Taniguchi Y (2013) The development of a large-area windowless energy dispersive X-ray detector for STEM-EDX analysis. Microsc. Microanal. 19, 1192-1193.

Zaluzec N J (2014) Analytical formulae for calculation of X-ray detector solid angles in the scanning and scanning/transmission analytical electron microscope. Microsc. Microanal. 20, 1318-1326.

Zhu Y, Inada H, Nakamura K, and Wall J (2009) Imaging single atoms using secondary electrons with an aberration-corrected electron microscope. Nat. Mater. 8, 808-812. 